\title{
Transmission of Data in Noisy Channel using Applications of Fountain Codes
}

\author{
Gurpreet Kour \\ Student \\ Electronics and Communication, \\ Vaishno Group of College,H.P
}

\begin{abstract}
Data is transmitted in the form of packets on the Internet. Due to various channel complexities, some packets may get lost and never reach their destination. Reliable transmission of data over the Internet is always desirable. Current networks that use mostly unicast -based protocols such as the transport control protocol TCP/IP and other unicast protocols place strong importance on the ordering of packets to simplify coding at the expense of increased traffic. An alternative approach is where packets are not ordered and the recovery of some subset of packets will allow for successful decoding. This class of such codes, called fountain codes, was pioneered by a startup called Digital Fountain and has greatly influenced the design of codes for binary erasure channels (BECs), a well-established model for the Internet [1]. This paper includes two fountain codes system models for packet recovery and bit error correction, a study of pre-codes of Raptor codes, and proposing a hybrid fast decoding algorithm to reduce the overheads of short and moderate-length LT codes.
\end{abstract}

\section{Keywords}

LT codes, Raptor codes, error rate.

\section{INTRODUCTION}

The conventional fountain codes work under important assumptions. All encoded packets accepted by a fountain decoder are assumed to be correct, because the system can use a forward error correction (FEC) scheme to correct the detected errors within a packet otherwise the packet will be discarded. The decoder constructs a generator matrix associated with these accepted symbols. Given a piece of data, consisting of $k$ symbols (which can be packets or bits), a fountain code produces a potentially limitless stream of output symbols with the properties that each output symbol is generated randomly (according to some distribution) and independently of every other symbol and it is possible to recover the original $k$ symbols from any set of $m$ received symbols, with high probability, for some $m$ (which is at least $k)$.

\section{FOUNTAIN CODES}

Fountain code produce output packets and send the packets via UDP rather than TCP. Fountain codes with efficient encoding and decoding algorithms with small overhead can be used in an excellent manner to solve the data distribution problems in upcoming $3 \mathrm{G}$ applications, such as rich-media transport. In fact, a version of fountain codes has been standardized in the 3GPPMBMS (multicast-broadcast multimedia services) standards body as the sole mandatory standard for data transport. Run a fountain encoder on each site. Since the symbols are produced randomly, symbols obtained from the different sites are indistinguishable and can be considered as coming from only one site. If a site breaks down or goes off-line for any reason, then this only leads to a slower download, as fewer output symbols are received. No extra management is necessary to ensure that the client has uninterrupted reception. Fountain codes are a class of codes designed for solving various data transmission problems, at the same time. Fountain codes with fast encoding and decoding algorithms, and (arbitrarily) small overhead are particularly interesting for solving these problems. Decoding delay of erasure codes, during pollution attack, and the index coding problem. While the use of erasure code improves the bandwidth performance of a broadcast network, it has a disadvantage of incurring a decoding delay. For example, for a client who has packet $\mathrm{c} 1$ and wants packets $\mathrm{c} 2$ and $\mathrm{c} 3$, coded packets $\mathrm{c} 2 \oplus \mathrm{c} 3$ and $\mathrm{c} 1 \oplus \mathrm{c} 2$ are both linearly independent with respect to $\mathrm{c} 1$, however only the latter coded packet can be instantly decoded by the client. Another problem related to security aspects of erasure codes is that of pollution attack. If the client admits even a single malicious coded packet from a malicious user, then during the decoding process, all the decoded packets will be corrupted. Traditional approaches to deal with system erasure are to use retransmission and replication techniques, which limits the reliability of the system, and adversely affects the throughput performance of the system. A series of Fountain codes - Tornado codes, LT codes, and Raptor codes - have been proposed, and patented, to address the decoding complexity. Thus the Fountain Codes are a new class of codes designed for robust, resynchronized, and scalable transmission of information from multiple senders to multiple receivers in a reliable manner over computer networks. The hypothesis of Fountain Codes is very exciting, and also provides new imminent into the theory of parity check codes. New asynchronous multicast applications using Fountain Codes is utilized by software simulation and hardware implementation.

\section{METHODOLOGY}

A class of codes is used to construct a robust and reliable transmission schemes. These codes are known as fountain codes. These codes can be decoded from a block with the maximum possible number of erasures. In this thesis work we are analyzing two applications of fountain codes: LT codes and Raptor codes. A transmitter having LT code uses a single code for efficient transmission in broadcast networks, where a single transmitter transferring a message to multiple receivers simultaneously over different channels. The transmitter with information message $\mathrm{u}$ consisting of $\mathrm{k}$ source symbols generates an infinite number of encoding symbols, which are broadcasted successively. The receiver is able to reconstruct the entire source reliably from any $\mathrm{k}$ received encoding symbols. If symbols are erased, an ideal fountain code receiver will just wait for $\mathrm{k}$ encoding symbols before reconstruct the information message. In this work following steps are made: 
1) We generate a matrix of an image with the help of image processing.

2) The transmitter with this information message consisting of $\mathrm{k}$ source symbols generates an infinite number of encoding symbols, which are broadcasted.

3) And the decoder uses the decoding process to decode the message. i.e, (Tanner graph of LT codes is used with is similar to tanner graph of LDPC codes) if there is at least one encoding symbol that has exactly one neighbor then the neighbor can be recovered immediately since it is a copy of the encoding symbol. The value of the recovered input is exclusive-ORed into any remaining encoding symbols that also have that symbol as a neighbor, the recovered input symbol is removed as a neighbor from each of these encoding symbols and the degree of each such encoding symbols is decreased by one to reflect this removal.

4) Then the BER performance is calculated in BEC and AWGN channel. The transmitter with information message $u$ consisting of $\mathrm{k}$ source symbols generates an infinite number of encoding symbols, which are broadcasted successively. The receiver is able to reconstruct the entire source reliably from any $\mathrm{k}$ received encoding symbols. If symbols are erased, an ideal fountain code receiver will just wait for $\mathrm{k}$ encoding symbols before reconstruct the information message.

\section{FLOWCHART}

This flow chart represents structure of the work done

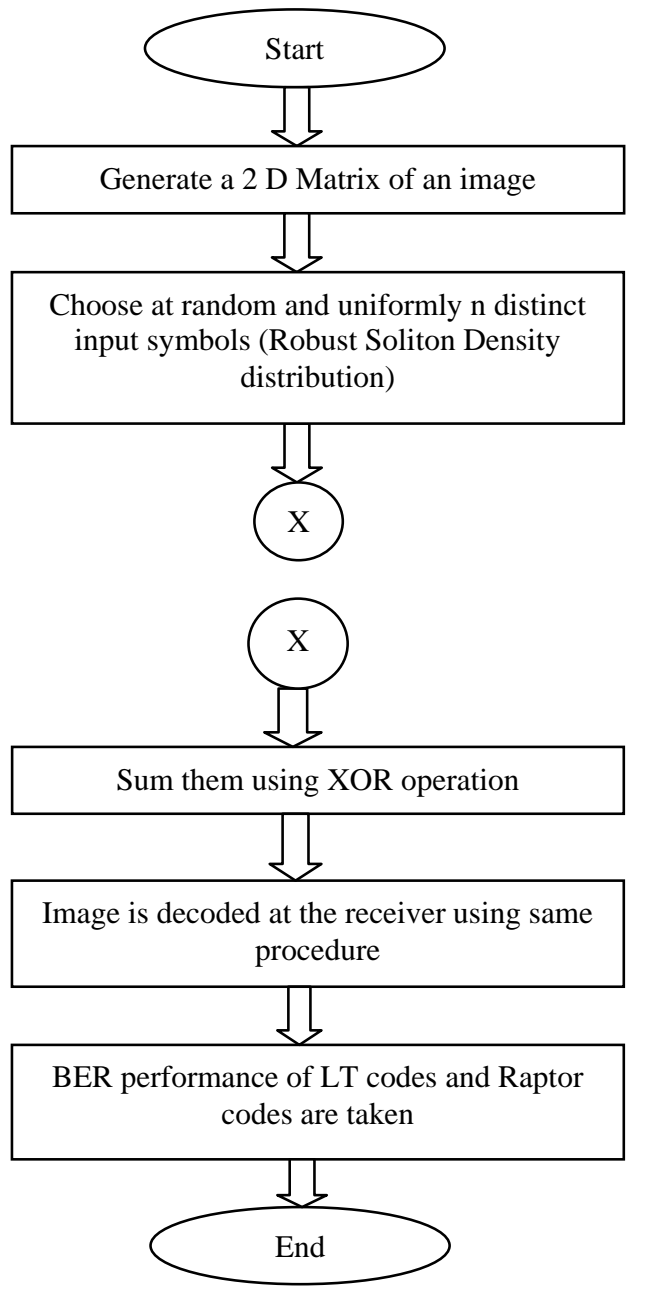

\section{RESULTS}

Fountain coding is used for improving the image transmission. This work is executed in following steps:STEP 1: The matrix of an image was generated with the help of image processing by selecting the image or by loading the image in the MATLAB. The image was a gray image in which each pixel has a range of 0 to 255 , the value of each pixel needs to use a byte to store, such as:

$$
\text { (232) } \mathrm{D}=(11101000) \mathrm{B}
$$

STEP 2: Encode the pixel information from the image. In the encoding process, data of each image has its own data group, the fountain codes were applied to each group and wait until after each group transport successfully and then restore the image pixel values, resulting in the recovered image. In the same way multiple images were loaded to form the matrix of images and recovered. STEP 3: In the next step, decoding of image was done using fountain (LT) decoding in order to recover the pixel information of the image and to find an error while transmission of an image.

Then, Decoding of image was done using fountain (Raptor) decoding in order to recover the pixel information of the image and to find an error while transmission of an image.

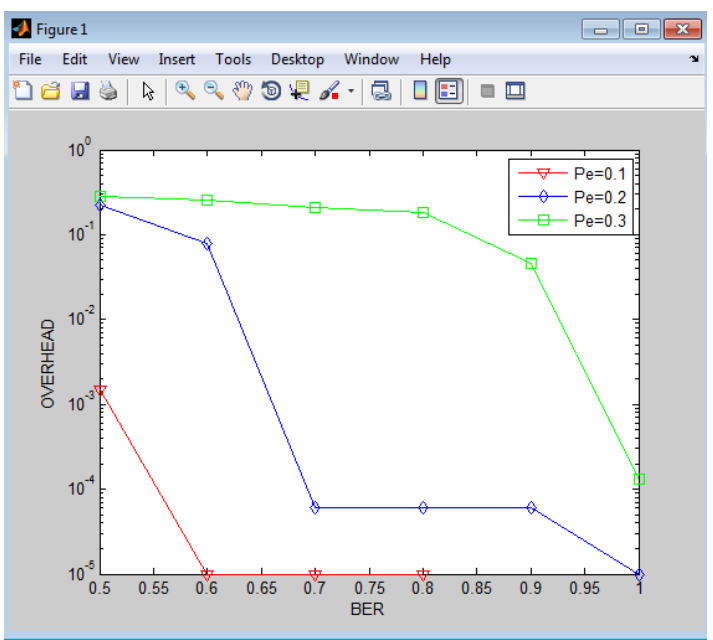

Figure 1: Graph was plotted for bit error rate vs overhead.

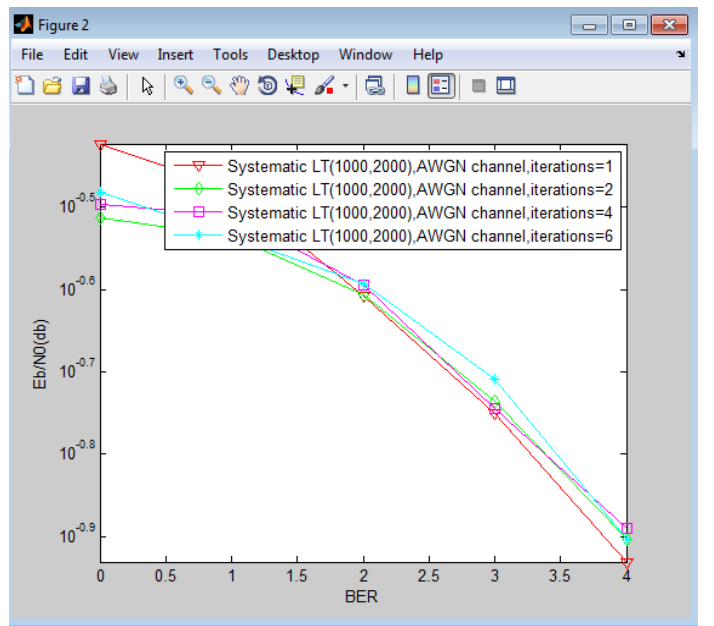

Figure 2: Output graph plotted for error Signal to noise ratio vs bit error rate 


\section{CONCLUSION}

Traditional approaches to deal with system erasure are to use retransmission and replication techniques, which limits the reliability of the system, and adversely affects the throughput performance of the system. A series of Fountain codes Tornado codes, LT codes, and Raptor codes - have been proposed and patented, to address the decoding complexity. Thus, the Fountain Codes are a new class of codes designed for robust, resynchronized, and scalable transmission of information from multiple senders to multiple receivers in a reliable manner over computer networks. The hypothesis of Fountain Codes is very exciting, and also provides new imminent into the theory of parity check codes. New asynchronous multicast applications using Fountain Codes is utilized by software simulation and hardware implementation. In this research work, it has been observed that the fountain (LT) coding provide more error rate while transportation of images and fountain (Raptor) coding provide less error rate while transmission.

\section{REFERENCES}

[1] Lin, S., and Costello, D.J. Jr.: "Error control coding. fundamentals and applications", Prentice-Hall, Englewood Cliffs, New Jersey, 1983.

[2] Luby M.: "LT codes", IEEE Symposium on Foundations of Computer Science, 2002, pp. 271-282.

[3] Shokrollahi A.: "Raptor codes", IEEE Transactions of information theory, 2006, pp. 2551-2567.

[4] Kushwaha H. and Chandramouli R.: "Reliable Multimedia Transmission Over Cognitive Radio Networks Using Fountain Codes", IEEE Transactions, DOI: 10.1109/JPROC.2007.909917

[5] Palanki R. and Yedidia J.S.: "Rateless Codes on Noisy Channels",Proceedings of the Conference on Information Sciences and Systems, 2004, pp.1-15.

[6] Sejdinovi'c D., Piechocki R., Doufexi A. and Ismail M.: "Fountain Code Design for Data Multicast with Side Information", IEEE Transaction on wireless communication, 2009, pp.5155-5165.

[7] Kasai K., Declercq D. and Sakaniwa K.: "Fountain Coding via Multiplicatively Repeated Non-Binary LDPC Codes”, IEEE Transactions on Communications, 2012, pp.2077-2083. 